\title{
BALLISTIC PERFORMANCE OF POROUS-CERAMIC, THERMAL PROTECTION SYSTEMS
}

\author{
J. E. Miller ${ }^{1}$, W. E. Bohl ${ }^{1}$, E. C. Christiansen ${ }^{2}$, B. A. Davis ${ }^{2}$, and C. D. Foreman ${ }^{3}$ \\ ${ }^{1}$ Lockheed Martin Space Systems Company, Denver CO 80127 \\ ${ }^{2}$ NASA Johnson Space Center, Houston TX 77058 \\ ${ }^{3}$ Lockheed Martin Exploration and Science, Houston TX 77058
}

\begin{abstract}
Porous-ceramic, thermal protection systems are used heavily in current reentry vehicles like the Orbiter, and they are currently being proposed for the next generation of US manned spacecraft, Orion. These systems insulate reentry critical components of a spacecraft against the intense thermal environments of atmospheric reentry. Additionally, these materials are highly exposed to space environment hazards like solid particle impacts. This paper discusses impact studies up to $10 \mathrm{~km} / \mathrm{s}$ on 8 $\mathrm{lb} / \mathrm{ft}^{3}$ alumina-fiber-enhanced-thermal-barrier (AETB8) tiles coated with a toughened-unipiece-fibrousinsulation/reaction-cured-glass layer (TUFI/RCG). A semi-empirical, first principals impact model that describes projectile dispersion is described that provides excellent agreement with observations over a broad range of impact velocities, obliquities and projectile materials. Model extensions to look at the implications of greater than $10 \mathrm{GPa}$ equation of state is also discussed. Predicted penetration probabilities for a vehicle visiting the International Space Station is $60 \%$ lower for orbital debris and $95 \%$ lower for meteoroids with this model compared to an energy scaled approach.
\end{abstract}

Keywords: Ballistic performance, porous, thermal protection system, hypervelocity impact.

PACS: 47.40.-x, 47.40.Nm.

\section{INTRO}

Porous-ceramic tiles insulate atmospheric reentry vehicles from reentry plasmas generated by atmospheric braking from orbital and exo-orbital velocities. Due to the necessity that these materials create a temperature gradient of approximately a thousand Kelvin over their thickness, it is important that these materials are near their pristine state prior to reentry. These tiles are also in general on exposed surfaces to space environment threats like orbital debris and meteoroids leaving a probability that these exposed surfaces will be below their prescribed values. Fortunately, owing to the typical small size of impact craters in these materials, the local flow fields over these craters afford some margin in thermal protection designs for these locally reduced performance values.
The acceptability of a locally reduced thermal protection system is limited with the key limit being a direct impingement of the reentry plasma on spacecraft structure. For regions of the vehicle that are subjected to the most intense reentry environments, this limit of acceptability can also be realized even if the structure is not directly exposed as the structural elements can be heated above their safe operating condition. The remaining thickness of insulating material after an impact is then an important parameter describing the worthiness of the vehicle to reenter. As such, the depth of penetration is the principal observable required when testing the performance of these materials to the orbital debris and meteoroid environments. In the study reported here, NASA's Hypervelocity Impact Test Facility (HITF) has impact tested these materials with projectiles 
typical of orbital debris and surrogate to meteoroids to determine the ballistic performance.

\section{EXPERIMENTAL PROCEDURE}

The tests performed are on target thermal protection materials typical of those used on the Orbiter and proposed for Orion. A density profile as a function of depth of a typical tile and substructure system is shown in Figure 2. From right to left, the figure shows the high density TUFI/RCG layer which tapers to the density of tile with its rear densification layer and the bonding pad and substrate to the extreme left. The TUFI/RCG layer provides a relatively high shock wave impedance material to push impacting materials to a higher pressure on impact. If the stresses are high enough to fragment/melt the projectile, the material decompresses extensively as it propagates in the highly porous tile resulting in scattered fragments and a diffusion of molten material. For threat particles too big to be arrested in the tile material, the densification and bonding layers' increased density provide an increased arresting potential prior to structural panel impact.

A pair of titanium powder enhanced orthogonal X-ray images of an off-normal impact crater is shown in Figure 1. The uppermost highcontrast plane in the views is the TUFI/RCG layer and the lowermost is the densification layer. The damage measurement normal to the TUFI/RCG layer is the maximum depth of penetration, and the damage measurements parallel to the TUFI/RCG layer are the widths. The product of the identified maximum depth of penetration and the tile density is the penetrated areal density value which can be

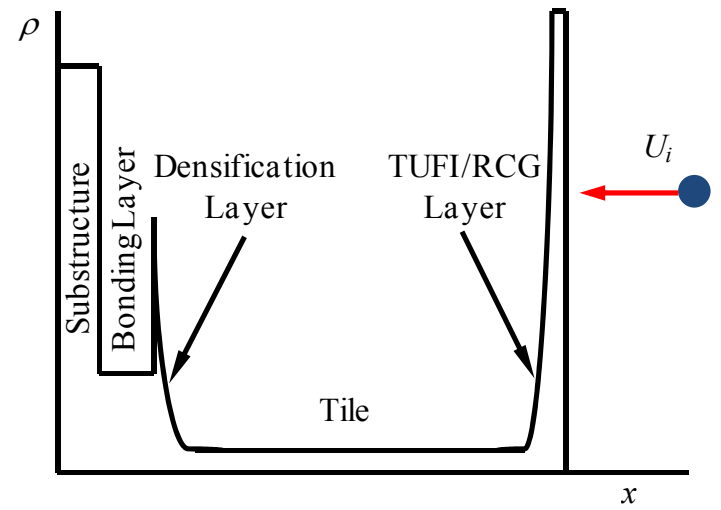

FIGURE 2. Density profile experienced by projectile.

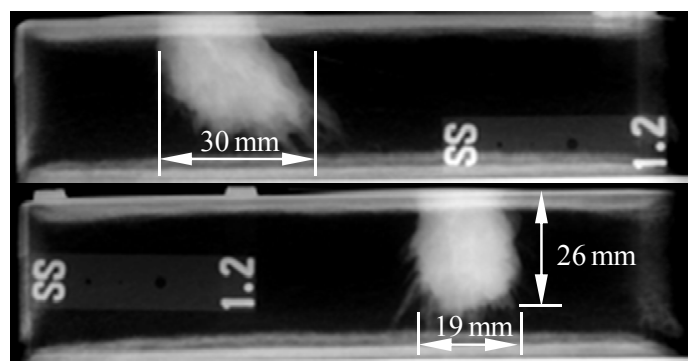

FIGURE 1. Parallel (top) and perpendicular (bottom) cross section of an impact crater with cavity dimensions.

used with the areal densities of the various layers of the thermal protection system.

This effort has performed 50 shots where the main projectile cavity is within the tile and minimal bonding pad damage has occurred. Nylon $^{\text {TM }}\left(1.14 \mathrm{~g} / \mathrm{cm}^{3}\right)$, aluminum 2017-T4 (2.796 $\left.\mathrm{g} / \mathrm{cm}^{3}\right)$ and steel $440 \mathrm{C}\left(7.68 \mathrm{~g} / \mathrm{cm}^{3}\right)$ projectiles have been considered. The penetrated cavity depth and widths are recorded for a variety of projectile sizes, impact obliquities, and impact speeds. The accuracy of the cavity measurements is limited to the resolution limit of the recording technique and to the limit of determining where cavities end. Using a fixed contrast ratio to define the cavity, the average measurement is used with the quadrature sum of the resolution limit and the variation about these averages for the measurement uncertainty. Along with the variable impact conditions, two areal densities of TUFI/RCG, $0.209 \pm 0.012$ and $0.158 \pm 0.009 \mathrm{~g} / \mathrm{cm}^{2}$, have been considered. For partial penetration of the rear densification layer, the depth of the cavity includes an equivalent tile thickness of the penetrated densification layer.

\section{RESULTS AND DISCUSSION}

It has been observed that porous materials, after passage of a strong shock wave, jump to about the density of the solid material. This property of a shock wave manifests in a linear relationship between the shock wave velocity and the particle velocity without a constant offset or $U=s u$ where $U, u$ and $s$ are the shock wave velocity, the particle velocity and proportionality constant, respectively. Using this kinematic approximation in the shock wave frame, the Lagrangian momentum response to hydrodynamic and mechanical forces is 


$$
\frac{1}{2} \frac{M}{s} \frac{d U^{2}}{d x}=-\left(\frac{1}{2} \frac{s+1}{s} \rho_{0} U^{2}+Y_{0}\right) A,
$$

where $x, M, \rho_{0}, Y_{0}$ and $A$ are the shock wave penetrated depth, the propagating mass, the density and compression yield strength of pristine tile $(\sim 0.8 \mathrm{MPa})$ and the effective area of the bow shock wave, respectively. This equation is similar to that developed in Ref. 1; however, the propagating mass is considered here as the projectile, the TUFI/RCG layer and a conic of accreted tile

$$
M=\left(\overline{m_{p}}+\overline{m_{T}}-\frac{\rho_{0} r_{p} / \omega}{3}\right) \pi r_{p}^{2}+\frac{\rho_{0} A x}{3}
$$

and the effective area is also depth dependent.

In Figure 3 the dependence of the ratio of half the cavity width to the penetrated depth for the normal impact records is shown as error ellipses. These expansion ratios are shown dependent on the ratio of an effective projectile areal density $\left(\overline{m_{p}}=4 / 3 r_{p} \rho_{p}\right.$ where $r_{p}$ and $\rho_{p}$ are the projectile radius and density, respectively) to TUFI/RCG areal density, $\overline{m_{T}}$. In this figure the cavity parameters are sorted by impact velocity bins with red, orange, green, blue and purple representing 4, 7, 8, 9 and $9.5 \mathrm{~km} / \mathrm{s}$. The curves shown are empirical fits using a modified logistics function

$$
\omega=\frac{0.3}{\operatorname{Exp}\left[\frac{\overline{m_{p}} U_{m}-\overline{m_{T}} U_{i}}{1 / 2 \overline{m_{T}} U_{m}}\right]+1}+0.2,
$$

where $U_{i}$ is the impact velocity and $U_{m}$ is a fit factor for this data set of $5.5 \mathrm{~km} / \mathrm{s}$. Nylon ${ }^{\mathrm{TM}}$ and steel projectiles have fit factors of $5.2 \mathrm{~km} / \mathrm{s}$ and 2.6 $\mathrm{km} / \mathrm{s}$, respectively. This function yields the required effective radius, $r=\omega x+r_{p}$, which in turn yields the effective area.

Transforming the penetrated depth in Eqn. 1 to the ratio of the effective to initial radius and normalizing by $1 / 2 \rho_{0} U_{0}^{2}$ results in

$$
\frac{1}{s}\left(\frac{\omega \mu-1 / 3}{\xi^{2}}+\frac{\xi-1}{3}\right) \frac{d \zeta}{d \xi}+\frac{s+1}{s} \zeta=-\psi
$$

where $\xi=r / r_{p}, \quad \zeta=U^{2} / U_{0}{ }^{2}, \quad \psi=2 Y_{0} / \rho_{0} U_{0}{ }^{2}$ and $\mu=\left(\overline{m_{p}}+\overline{m_{T}}\right) / \rho_{0} r_{p}$. The term $U_{0}$ is the initial shock wave velocity in the tile after release from the TUFI/RCG and derived from Eqn. 1

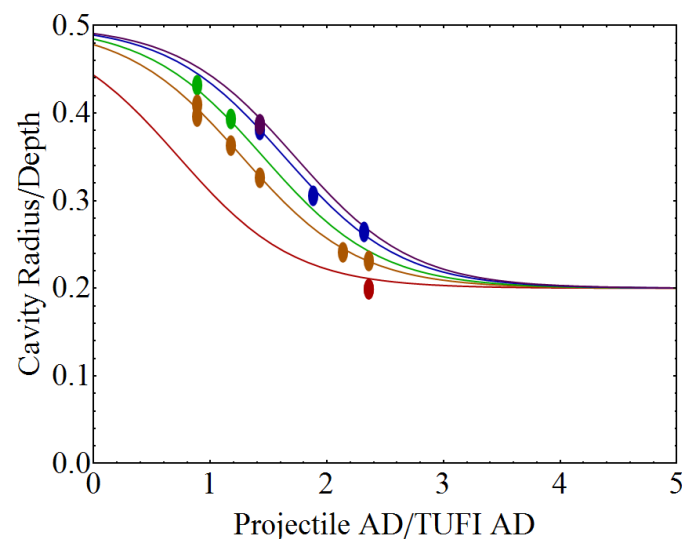

Figure 3. Dependence of aluminum impact radius to depth on projectile to TUFI/RCG for 4 (red), 7 (orange), 8 (green), 9 (blue) and 9.5 (purple) $\mathrm{km} / \mathrm{s}$

$$
U_{0}=s U_{i}\left(\frac{\overline{m_{p}}}{\overline{m_{p}}+\overline{m_{T}}}\right)^{\frac{s_{T}+1}{2}}
$$

where $s_{T}$ is the TUFI/RCG layer shock wave slope.

The projectile and the swept tile mass are completely stopped when the dimensionless parameter for velocity goes to zero, thus the root of the function is the final non-dimensional radius. Transformation of these roots to projectile mass results in Figure 4. This figure represents the performance of this model relative to the collection of aluminum, Nylon ${ }^{\mathrm{TM}}$ and steel shots for all impact obliquities. To accommodate for the impact obliquity effects, a cosine of the impact obliquity term is used with the projectile density dependent powers of $-2 / 3$ for Nylon ${ }^{\mathrm{TM}},-8 / 3$ for aluminum and -3 for steel. The dominate uncertainties in the abscissa are the penetrated areal density, $m_{f}$, and the expansion ratio which are related to the cavity.

The model's predicted ballistic performance for a 1.5 " tile with a $0.209 \mathrm{~g} / \mathrm{cm}^{2}$ TUFI/RCG coating (heavyweight) is shown in Figure 5 in solid blue along with the ballistic performance for equal thickness tiles using $0.158 \mathrm{~g} / \mathrm{cm}^{2}$ (lightweight TUFI/RCG) and $0.044 \mathrm{~g} / \mathrm{cm}^{2}$ (RCG only) coatings in solid green and red, respectively. In all three cases, projectile sizes below the respective curves are not expected to fully penetrate the tile while projectile sizes above the curves are expected to penetrate the tile. Due to the longer duration at high stress states, the onset of fragmentation and melt with heavyweight TUFI/RCG is achieved at 


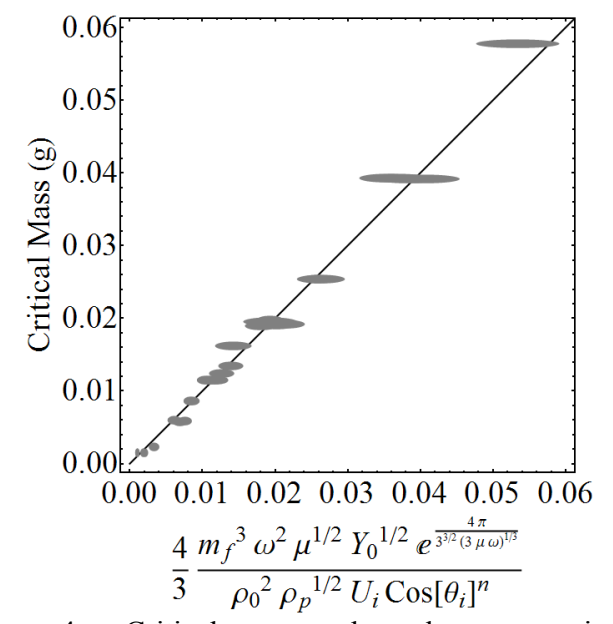

Figure 4. Critical mass dependence on impact parameters with impact data represented by error ellipses and exact model agreement on the curve.

lower impact velocities than with lightweight TUFI/RCG and RCG only layers.

The shock wave compression is an important parameter to the ultimate ballistic performance of the tile. It has been shown that shock wave compression conditions transition between two different nearly constant values [2]. For lower shock wave strengths the material compresses to near the crystalline density, and at high shock wave strengths the material compresses to only a few times the initial density. This behavior has been incorporated in this impact model using a

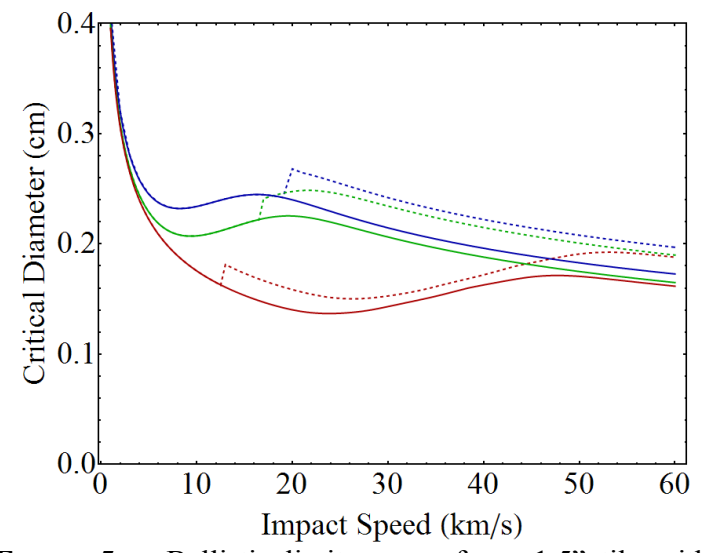

Figure 5. Ballistic limit curves for a 1.5" tile with heavyweight TUFI/RCG (blue) and equal thickness of lightweight TUFI/RCG (green) and RCG (red) surface coatings. Solid curve is compression to near-crystalline density and dashed is six-fold compression at $10 \mathrm{GPa}$. piecewise function for $s$ on shock wave velocity where $s$ is taken as 1.2 (six-fold compression) for shock wave velocities above $9 \mathrm{~km} / \mathrm{s}$ and 1.055 ( $\sim$ nineteen-fold compression) for velocities below, which is similar to the behavior shown in Ref. 4. The ballistic performance of the 1.5 " tile with this behavior is shown as dashed curves in Figure 5 with the same coloring. As can be seen, incorporating dissociation/ionization in the model predicts an increased performance.

To determine the impact of these observations on spacecraft risk assessments, a comparison of the ballistic performance at a depth of $2.7 \mathrm{~cm}$ from this model and a traditional energy scaled model like that of Ref. 4 are used. Using these models with the solid particle environments for the International Space Station (ISS) indicates a $60 \%$ lower predicted probability of orbital debris penetrating to $2.7 \mathrm{~cm}$ in a tile with a lightweight TUFI/RCG coating. Due to the higher expected impact velocities of meteoroids, the predicted probability of penetration to the same depth is $95 \%$ lower.

\section{CONCLUSIONS}

Using the data obtained, a model using the material equation of state and strength properties of AETB8 tiles are used to develop a model that explains these findings and facilitates the further extrapolation to alternative tile configurations and impact conditions. Further work is necessary to develop analytical models of the projectile interaction with the discrete surface coatings of the tile and the obliquity effects, along with, further studies and work is necessary to extend the model to determine the impact of higher shock wave impedance at $>10 \mathrm{GPa}$ on ballistic performance.

\section{REFERENCES}

1. Trucano, T. G. and Grady, D. E., "Impact Shock and Penetration Fragmentation in Porous Media," Int. J. of Imp. Eng., 17, 4-6, 1995, pp. 861-872.

2. Boehly, T. R., et al., "Measurements of the Release of Alpha Quartz: A New Standard for ImpedanceMatching Experiments," in Shock Compression of Condensed Matter, 2007 (Elert, Furnish, Chau, Holmes, Nguyen eds.), part I, pp. 19-22.

3. Christiansen, E. L., and Freisen, L., "Penetration Equations for Thermal Protection Materials," Int. J. of Imp. Eng., 20, 1-5, 1997, pp. 153-164. 


\section{Referee Material}

Equation 1 represents a Langrangian form of the combination of the continuity, a simplified kinematic form of the equation of state and the momentum conservation equation. Starting with the momentum conservation equation in a frame at rest with the particle along the propagation direction

$$
\frac{d M u}{d t}=-F_{H}-F_{M}
$$

the left-hand side is the momentum changes experienced by the projectile and the target material, and the righthand side is the sum of forces acting on the projectile. The mass within the control volume, $M$, includes the compressed material and the projectile. The kinematic form of the equation of state is $U=s u$ where $s$ is the linear relationship between the particle velocity, $u$, and the shock wave velocity, $U$, assuming near zero sound speed. With this equation of state the hydrodynamic (differential pressure between shock wave front surface and vacuum surface) is given by:

$$
F_{H}=\rho_{0} U^{2} / s A \text {. }
$$

The mechanical (resistance to compression) force is given by:

$$
F_{M}=Y_{0} A \text {. }
$$

The terms $\rho_{0}, Y_{0}$ and $A$ are the initial density of the tile, the compressive yield strength of the tile and the projected area of the bow shock wave, respectively. The mass within the control volume is a summation of the mass of the projectile, TUFI/RCG layer and the accreted tile mass given by:

$$
M=\left(\overline{m_{p}}+\overline{m_{T}}-\frac{\rho_{0} r_{p} / \omega}{3}\right) \pi r_{p}^{2}+\frac{\rho_{0} A x}{3}
$$

where $\overline{m_{p}}$ and $\overline{m_{T}}$, are the effective areal density of the spherical projectile $\left(4 / 3 \rho_{p} r_{p}\right.$ where $\rho_{p}$ and $r_{p}$ are the projectile density and radius) and the TUFI areal density. The second term is the mass of the tile that has directly undergone accelerations by the expanding shock wave front from the projectile which is the product of the density of the tile and the volume of the cone created by the expansion where $r_{p} / \omega$ removes the volume that is extrapolated beyond the TUFI/RCG layer interface and $\mathrm{w}$ is from Eqn. 2. Transforming the left-hand side to Lagrangian coordinates and expanding yields:

$$
\frac{d M u}{d t}=\frac{M}{s} \frac{d U^{2} / 2}{d x}+U^{2}\left(1-\frac{1}{2} \frac{\rho}{\rho_{0}}\left(\left(\frac{1}{s}\right)^{2}-1\right)\right) \frac{d M}{d x}
$$

where the control volume advances at the shock wave velocity. The first term in the expanded equation is the reaction of the control volume to the applied forces, and the second term is the momentum transfer to the accreted mass. The term $\frac{1}{2} \frac{\rho}{\rho_{0}}\left(\left(\frac{1}{s}\right)^{2}-1\right)$ transforms the accreted mass from the control volume to the frame at rest with the projectile accounting for the non-isentropic effects that occur within the shock front. Combining the expanded Lagrangian form of the momentum equation with the forces experienced by the advancing control volume on a per unit area basis yields

$$
\frac{1}{2}\left(\left(\overline{m_{p}}+\overline{m_{T}}-\frac{\rho_{0} r_{p} / \omega}{3}\right) \frac{r_{p}^{2}}{r^{2}}+\frac{\rho_{0} x}{3}\right) \frac{1}{s} \frac{d U^{2}}{d x}+\rho_{0} \frac{s+1}{s} \frac{U^{2}}{2}=-Y_{0}
$$


where the simplified tile equation of state property $\frac{\rho}{\rho_{0}}=\frac{s}{s-1}$ is used. Using the projected radius equation $r=\omega x+r_{p}$ to transform the independent variable to correspond to the initial condition and dividing through by $1 / 2 \rho_{0} U_{0}^{2}$ one arrives at

$$
\frac{1}{s}\left(\frac{\omega\left(\overline{m_{p}}+\overline{m_{T}}\right) / \rho_{0}-r_{p} / 3}{\left(r / r_{p}\right)^{2}}+\frac{r-r_{p}}{3}\right) \frac{d U^{2} / U_{0}^{2}}{d r}+\frac{s+1}{s} \frac{U^{2}}{U_{0}{ }^{2}}=-\frac{Y_{0}}{1 / 2 \rho_{0} U_{0}{ }^{2}}
$$

where $U_{0}$ is the velocity of the projectile as it breaks through the TUFI/RCG layer into the low density tile.

Equation 4 is the non-dimensional form of A7 when $r$ is transformed to $\xi=r / r_{p}$ given by

$$
\frac{1}{s}\left(\frac{\omega \mu-1 / 3}{\xi^{2}}+\frac{\xi-1}{3}\right) \frac{d \zeta}{d \xi}+\frac{s+1}{s} \zeta=-\psi
$$

where $\zeta=U^{2} / U_{0}{ }^{2} \mu=\left(\overline{m_{p}}+\overline{m_{T}}\right) /\left(\rho_{0} r_{p}\right)$ and $\mu=Y_{0} /\left(1 / 2 \rho_{0} U_{0}^{2}\right)$. This first-order differential has the solution

$$
\zeta=-\frac{s}{s+1} \psi+\left(1+\frac{s}{s+1} \psi\right) \frac{\left.f\right|_{\xi=1}}{f}
$$

where $f$ is given by

$$
f=\operatorname{Exp}\left[(s+1) \int \frac{d \xi}{\frac{\omega \mu-1 / 3}{\xi^{2}}+\frac{\xi-1}{3}}\right] .
$$

The general solution for $f$ is given by

$$
f=\prod_{i}\left(\xi-a_{i}\right)^{\frac{3(s+1) a_{i}}{3 a_{i}-2}}
$$

where $a_{i}$ are the three roots of the equation

$$
k^{3}-1-\xi^{2}+\xi^{3}=0,
$$

where $k=(3 \omega \mu)^{1 / 3}$. The combination of A9, A11 and A12 is the full analytic solution to Eqn. 4. For the case that $k^{3}$ is large, these roots are approximately given by

$$
a_{i} \approx \frac{1}{3}+(-1)^{\frac{16}{3} i-1} k
$$

where $i=1,2,3$.

Performing an asymptotic expansion of the simplified form of A11 at the initial condition $\xi=1$ for $k$ greater than one, the initial condition integral is given approximately by

$$
\left.f\right|_{\xi=1} \approx\left(k-\frac{4 \pi}{9 \sqrt{3}}\right)^{3(s+1)} .
$$



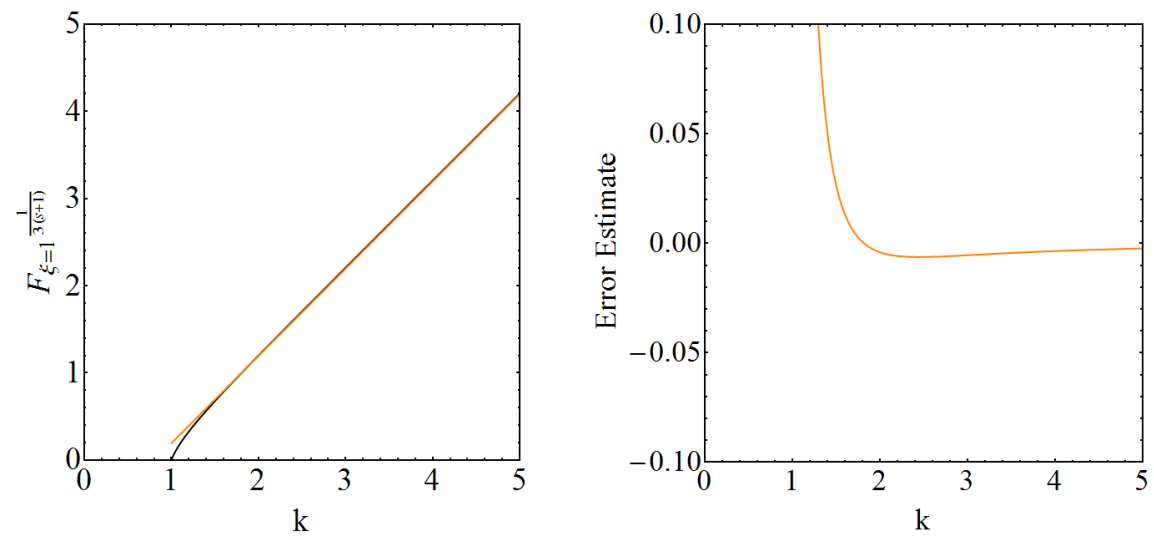

FIGURE 6. Initial condition approximation (orange) relative to full numerical integral (black) as a function of the TUFI/RCG parameter and the error of estimate for the initial condition integral.

The dependence of the $3(s+1)$ root of this approximate initial condition is shown in Figure 6 (orange curve) as a function of $k$ relative to the full numerical solution of A11 (black curve). As can be seen in the Figure 6 the function is reasonably approximated to within a few percent to this simple linear relationship for values of $k$ in the range of 2.5 to 4.5 which is typical of the aluminum impacts discussed in Figure 6 with the predominant value of $k$ being at about 3.5. Similarly in Figure 7, an asymptotic expansion at the condition $\xi \gg k>1$, yields the integral dependence

$$
\left.f\right|_{\xi \gg k>1} \approx(\xi-1)^{3(s+1)} .
$$

This approximation (orange curve) along with the full numerical solutions of A11 for $k$ (gray curves) equal to 2.5, 3.5 and 4.5 (light, medium and dark, respectively) are shown along with the errors of estimate for $k$ equal to 2.5, 3.5 and 4.5 (light, medium and dark orange, respectively). Again it can be seen that this approximation is good for the typical $\xi>10$ expansions of Figure 7. Substituting these approximations into A9 one obtains
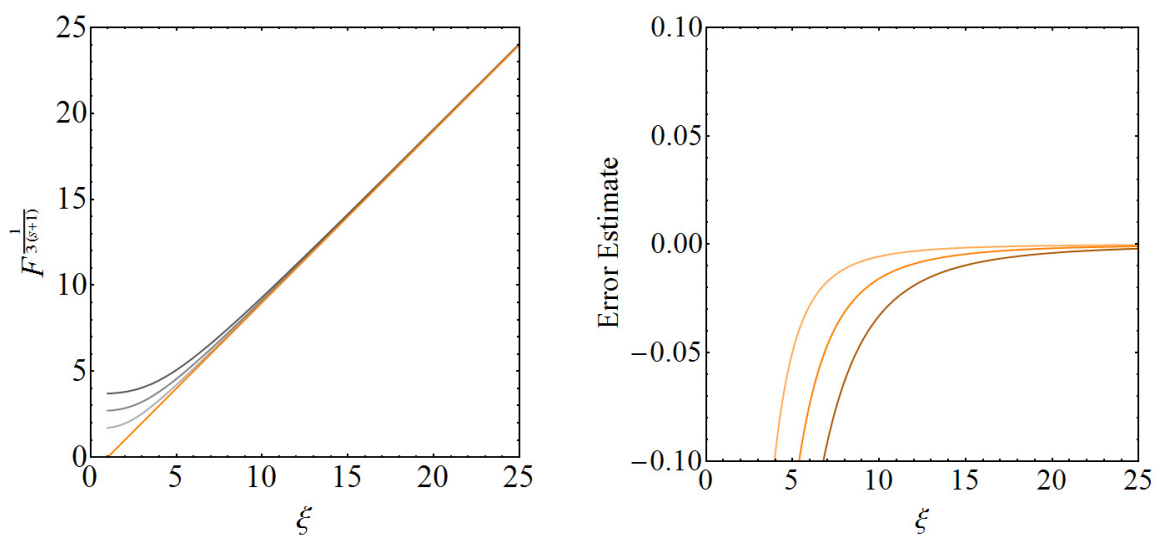

FigURE 7. Final condition approximation (orange) relative to full numerical integral for $\mathrm{k}$ equal to 2.5 (light gray), 3.5 (medium gray) and 4.5 (dark gray) as a function of the expansion and the error of estimate for $\mathrm{k}$ equal to 2.5 (light orange), 3.5 (medium orange) and 4.5 (dark orange) on the final condition integral. 


$$
\zeta \approx-\frac{s}{s+1} \psi+\left(1+\frac{s}{s+1} \psi\right)\left(\frac{k-\frac{4 \pi}{9 \sqrt{3}}}{\xi-1}\right)^{3(s+1)}
$$

For the case where the projectile stops, $\xi_{f}, \zeta$ goes to zero or in terms of $\xi_{f}$

$$
\xi_{f}-1 \approx\left(k-\frac{4 \pi}{9 \sqrt{3}}\right)\left(\frac{1+\frac{s}{s+1} \psi}{\frac{s}{s+1} \psi}\right)^{\frac{1}{3(s+1)}} .
$$

Under the impact conditions considered here, $\psi$ is much smaller than 1 allowing the numerator in the root to be approximately 1 . Using the definition of $\xi$ and solving for the projectile radius on the left-hand side yields

$$
r_{p} \approx \frac{\omega x_{f}}{(3 \omega \mu)^{1 / 3}} \operatorname{Exp}\left[\frac{4 \pi}{9 \sqrt{3}} \frac{1}{(3 \omega \mu)^{1 / 3}}\right]\left(\frac{s}{s+1} \frac{Y_{0}}{1 / 2 \rho_{0} U_{0}^{2}}\right)^{\frac{1}{3(s+1)}}
$$

where the dimensionless parameter definitions are substituted back with the exception of the dimensionless reduced mass. A Taylor expansion approximation is substituted for the small but not negligible constant. Converting to the mass of the impactor

$$
m_{p} \approx \frac{4 \pi}{9} \frac{\rho_{p} \omega^{2} x_{f}^{3}}{\mu} \operatorname{Exp}\left[\frac{4 \pi}{3^{3 / 2}} \frac{1}{(3 \omega \mu)^{1 / 3}}\right]\left(\frac{s}{s+1} \frac{Y_{0}}{1 / 2 \rho_{0} U_{0}^{2}}\right)^{\frac{1}{s+1}} .
$$

Using Eqn. 5 for $U_{0}$ and substituting the definition of the reduced mass, $\mu$, for the effective projectile and TUFI/RCG areal densities the relationship between the initial condition velocity and the impact velocity $U_{i}$ is given by

$$
U_{0} \approx U_{i}\left(\frac{4}{3} \frac{\rho_{p}}{\rho_{0} \mu}\right)^{\frac{1+s_{T}}{2}}
$$

Substituting the approximation that the shock wave velocity is the particle velocity in the tile and the initial condition velocity into A19 yields

$$
m_{c} \approx \frac{4 \pi}{9} \frac{m_{f}^{3} \omega^{2} \rho_{p} Y_{0}{ }^{1 / 2}}{\rho_{0}{ }^{7 / 2} \mu U_{i}} \operatorname{Exp}\left[\frac{4 \pi}{3^{3 / 2}} \frac{1}{(3 \omega \mu)^{1 / 3}}\right]\left(\frac{3}{4} \frac{\rho_{0} \mu}{\rho_{p}}\right)^{\frac{1+s_{T}}{2}} .
$$

Noting that the value of $s_{T}$ is typically about 2 for a compression of an approximate $1.3 \mathrm{~g} / \mathrm{cm}^{3} \mathrm{TUFI} / \mathrm{RCG}$ layer one arrives at the simplified transcendental equation

$$
m_{c} \approx \frac{\pi}{2 \sqrt{3}} \frac{m_{f}^{3} \omega^{2} Y_{0}^{1 / 2} \mu^{1 / 2}}{\rho_{0}^{2} \rho_{p}{ }^{1 / 2} U_{i}} \operatorname{Exp}\left[\frac{4 \pi}{3^{3 / 2}} \frac{1}{(3 \omega \mu)^{1 / 3}}\right],
$$

which represents a $20 \%$ difference from the lead coefficient of $4 / 3$ in Figure 4. 